\title{
Synthesis of few-wall carbon nanotubes using methanol/ propanol mixture by chemical vapour deposition
}

\author{
Abdulrazzak $\mathrm{FH}^{* *}$, Abbas $\mathrm{AM}^{2}$ and Hussein $\mathrm{FH}^{3}$ \\ ${ }^{1}$ Department of Chemistry, College of Education for Pure Science, Diyala University, Iraq \\ ${ }^{2}$ Department of Chemistry, College of Education for Pure Science, Baghdad University Iraq \\ ${ }^{3}$ Department of Pharmaceutics, College of Pharmacy, Babylon University, Iraq
}

\begin{abstract}
Carbon nanotubes with few walls are highly appreciated for specific physiochemical properties and technological with huge applications. In this paper, we present an alternative process that can lead to lowering the manufacturing in time and cost. The process of synthesized were done by chemical vapor deposition with homemade portable tube furnace reactor. Methanol/ Propanol mixture is utilized as a source of carbon on silica surface caver with carbon black (CB) as support for growth CNTs at two temperature 450 and $750^{\circ} \mathrm{C}$. The characterization of FWNTs was performed by X-Ray diffraction, Raman spectroscopy, transmission electron microscopy TEM, Brunauer, Emmett and Teller BET and Thermalanalysis TGA. The main interest of this study are to give the basis of an efficient simple synthesis process to produce CNTs of few walls for removing pollutant by adsorption. The method was succeeded to synthesize FWNT450 which more surface area and more active site than FWNT750 in adsorption properties.
\end{abstract}

\section{Introduction}

The process of synthesized and purified nanomaterials represent a large challenge due to the cost which is still higher than the micro materials. Large efforts to manufacture nanomaterials for their enormous applications such nano-adsorbents which used to remove pollutants from environmental with high activity and sensitivity [1]. Carbon nanomaterials CNMs one of the new nanomaterials entered the science with all forms such fullerene and tubular structures CNTs [2]. The extraordinary physiochemical properties and less toxicity for carbon nanotubes then fullerene encourage laboratories in all world to study and try to take advantage of these structures [3]. Generally, carbon nanotubes include two form: mono layers tubes or single wall carbon nanotubes SWNTs and multi-layer tubes MWNTs [4]. Recently, few-walled carbon nanotubes FWNTs new class refer to double or triple sheet until seven graphene sheets [5]. They can be considered as an intermediate structure and properties with cost between SWNTs and MWNTs. Arc-discharge, laser ablation, and chemical vapor deposition CVD represent the primary techniques for synthesis CNTs [5]. Chemical vapor deposition is simple and law cost with less complicated as compare with the ether techniques [5]. Many hydrocarbon materials are use as sources of carbon to synthesized CNTs such Methane acetylene, benzene and ethanol [6-9]. Methanol mostly used with the mixture to synthesized CNTs Due to produce high ratios of unconverted carbon, but enhancement effect of the $\mathrm{OH}$ radical which evolves from dissociation encourage to use it $[4,10]$. Vander et al. [11] suggested that catalytic behavior can be explained by electron donation to the support when creating d-vacancies, which acts as an active site to exit the hydrocarbon dissociation. Reilly and Whitten [12] completed this view when assuming that the donor or accept electrons enhance the formation and condensation of free radicals. During the pyrolysis of hydrocarbon, the bonds will be broken between the carbon and other atoms to produce different fragments of free radicals allowing for the rapid rearrangement of carbon bonds. The yield of CNTs mostly reduced under the presence of oxygen atoms, on the precursor molecule, with high-temperature oxidation is possible [13,14]. Mitri et al. [15] and Rajard et al. [16] studied the effects of temperature on the synthesis of carbon nanotubes. Both two groups confirmed to important facts which should be chosen the optimum temperature for growth specific crystalline, when estimates by finding the ratios $I_{D} /$ $\mathrm{I}_{\mathrm{G}}[15,16]$. Use of natural resources and development of reactors are taken as advantageous for the research. Many literatures were reported preparing CNTs from natural sources of modifying CVD method. Nady [17] use rice straw as waste materials and source of carbon with different ratios of ferrocene /Ni. Kure et al. [18] reported modified commercial microwave ovens with quartz tubes to synthesized CNTs from Polyethylene. Gonzalez et al. [19] doping carbon nanotubes with Nitrogen from Pyridine/ ferrocene as sources of carbon and catalyst respectively, by modified chemical vapor deposition method using a novel two steps thermal technique. Many literatures such mentions previously, include modified strategy for preparation of complicating causing to increase the cost of preparing in addition to pre-treatment for sources of carbon before use. In this work, we use simple homemade portable reactor to synthesized FWNTs from methanol/ propanol as sources of carbon which is cheap and available in the chemical and physical labs. The change in temperature with an effect of free radical was applied to synthesize two kinds FWNTs with various on active site and surface area, which act perfect nano adsorbent materials. The Congo Red as an absorbent substance was chosen to study the activities of adsorption for both synthesized FWNTs.

Correspondence to: Firas H Abdulrazzak, Department of Chemistry, College of Education for Pure Science, Diyala University, Iraq; Tel: +9647702400280; E-mail: firas_habeb2000@yahoo.com

Key words: FWNT, Black Carbon, Defect, TEM, Adsorption

Received: August 02, 2017; Accepted: September 15, 2017; Published: September 18,2017 


\section{Experiment}

\section{Materials}

The material used in this experiment was methanol (99.93\%) from Alfa Assar and propanol ( $>99 \%)$ from Sigma-Aldridge which used without further purification. Black carbon was purchase from SigmaAldrich with average pore diameter $100 \AA \pm 10 \AA$ and purities $>99.95 \%$. The Hydrogen peroxide $\mathrm{H}_{2} \mathrm{O}_{2}$ was purchase from Barcelona, Spain in $30 \%$ percent weight. Congo Red (CR), $\mathrm{C}_{32} \mathrm{H}_{22} \mathrm{~N}_{6} \mathrm{Na}_{2} \mathrm{O}_{6} \mathrm{~S}_{2}$, with formal weight equal to 696, were supplied by from Merck.

\section{Synthesis of MWNTs}

The Few-wall carbon nanotubes FWNTs were prepared by chemical vapor deposition method CVDs as shown in Figure 1 with homemade tube furnaces.

Briefly, the tube furnace consist of two tubes which made from ceramic: the inner tubes with length $120 \mathrm{~cm}$ and diameter $3 \mathrm{~cm}$ when the precipitation was an accord. The outer tube was surround to the inner tube with $7 \mathrm{~cm}$ in diameter and $100 \mathrm{~cm}$ in length. The length of the thermal cable was $40 \mathrm{~cm}$, with an ability to control the temperature up to $800^{\circ} \mathrm{C}$ within $\pm 2^{\circ} \mathrm{C}$. The carbon black was used to complete removing the air and the remains act as catalyst for growth CNTs. The black carbon were dissolved in $100 \mathrm{ml}$ of ethanol then dropped inside inner tube. Before switching on the furnace, nitrogen gas was purged to remove the air from all the tube reaction with flow rate $125 \mathrm{~cm}^{3} / \mathrm{min}$. The furnace was switched on and heated to the reaction temperature. The synthesis was conducted at $450^{\circ} \mathrm{C}$ at atmospheric pressure, with a typical reaction time $30 \mathrm{~min}$ in a nitrogen atmosphere and a flow of $125 \mathrm{~cm}^{3} / \mathrm{min}$. When the furnace attained the desired temperature the $\mathrm{N}_{2}$ gas flow was reduced slowly to a rate of $80 \mathrm{~cm}^{3} / \mathrm{min}$. The $\mathrm{N}_{2}$ gas carried the sources of carbon when passing through a vaporizer of the mixture (1:1) methanol/propanol which evaporate at $70^{\circ} \mathrm{C}$. After deposition, the furnace was switched off and allowed to cool down at room temperature under a continuous $\mathrm{N}_{2}$ flow. The CNTs that produces from this reaction was labeling FWNT450. The same conditions were done in preparing the second types of FWNTs only change the temperature to $750^{\circ} \mathrm{C}$ and labeling FWNT750. The product was collected to characterized then purify for adsorption process.

\section{Purification process}

The process of purification was done as we reported on our previous work without treatment of $5 \% \mathrm{HNO}_{3}$ [20]. Briefly, (125 mg) of asprepared FWNT450 and FWNT750 were dispersed with an ultrasonic water bath in 150 ethanol alcohol for 10 min then using separation funnel, to re-distribution the aggregated particles. The lower part was separated and dispersion with $100 \mathrm{ml}$ of hydrogen peroxide $\mathrm{H}_{2} \mathrm{O}_{2}$ at $15^{\circ} \mathrm{C}$ for $12 \mathrm{~h}$. The solution was diluted by adding $100 \mathrm{ml}$ distilled water then allowed to reach room temperature. The mixture was heating gradually to $50^{\circ} \mathrm{C}$ for 30 minutes to complete dissociation most of the hydrogen peroxide. Finally, the sample was filtered and washed by distil-water and dried at $100^{\circ} \mathrm{C}$ for $12 \mathrm{~h}$.

\section{Results and discussion}

\section{Characterization}

The X-ray diffraction (XRD) patterns were done on a (Riga Rotalflex) (RU-200B) X-ray diffractometer using $\mathrm{Cu} \mathrm{Ka}$ radiation (wavelength $0.15405 \mathrm{~nm}$ ) with a Ni filter. The tube current was $100 \mathrm{~mA}$ with a tube voltage of $40 \mathrm{kV}$. The $2 \theta$ angular regions between 10 and $80^{\circ}$ were explored at a scan rate of $5 \%$ min. For all the XRD analysis, the resolution in the $2 \theta$ scans was kept at $0.02^{\circ}$. Raman analysis was done by using Sentara infinity 1 Broker with using laser light at 530 $\mathrm{nm}$, intensity $2 \mathrm{~m} \mathrm{~W}$ for 5 lops per $2 \mathrm{~s}$ and resolution equal to $3-5 \mathrm{~cm}$. TEM images were performed in a Jeol JEM 2100 operating at $200 \mathrm{kV}$ and equipped with a LaB6 filament, and an EDX Broker X-Flash 5030 detectors. The BET surface area was measured by using Micrometric Auto MATE 23. Thermalanalysis was conducted using a Thermal Gravimetric Analysis system (TGAQ 500 U20.13 Build 39) equipped with a high temperature furnace and a Type-S sample carrier. The TGA experiments were performed by heating from room temperature to $900^{\circ} \mathrm{C}$ at a heating rate of $10^{\circ} \mathrm{C} / \mathrm{min}$ within $50 \mathrm{ml} / \mathrm{min}$ of dry flowing air.

The process of synthesized was done on the surface of silica covered with black carbon. As-prepared FWNTs, include impurities which mostly represent by amorphous carbon, graphite, and unconverted carbon. Actually, the predicted in impurities can be related to conditions of precipitation which made on the support without using a

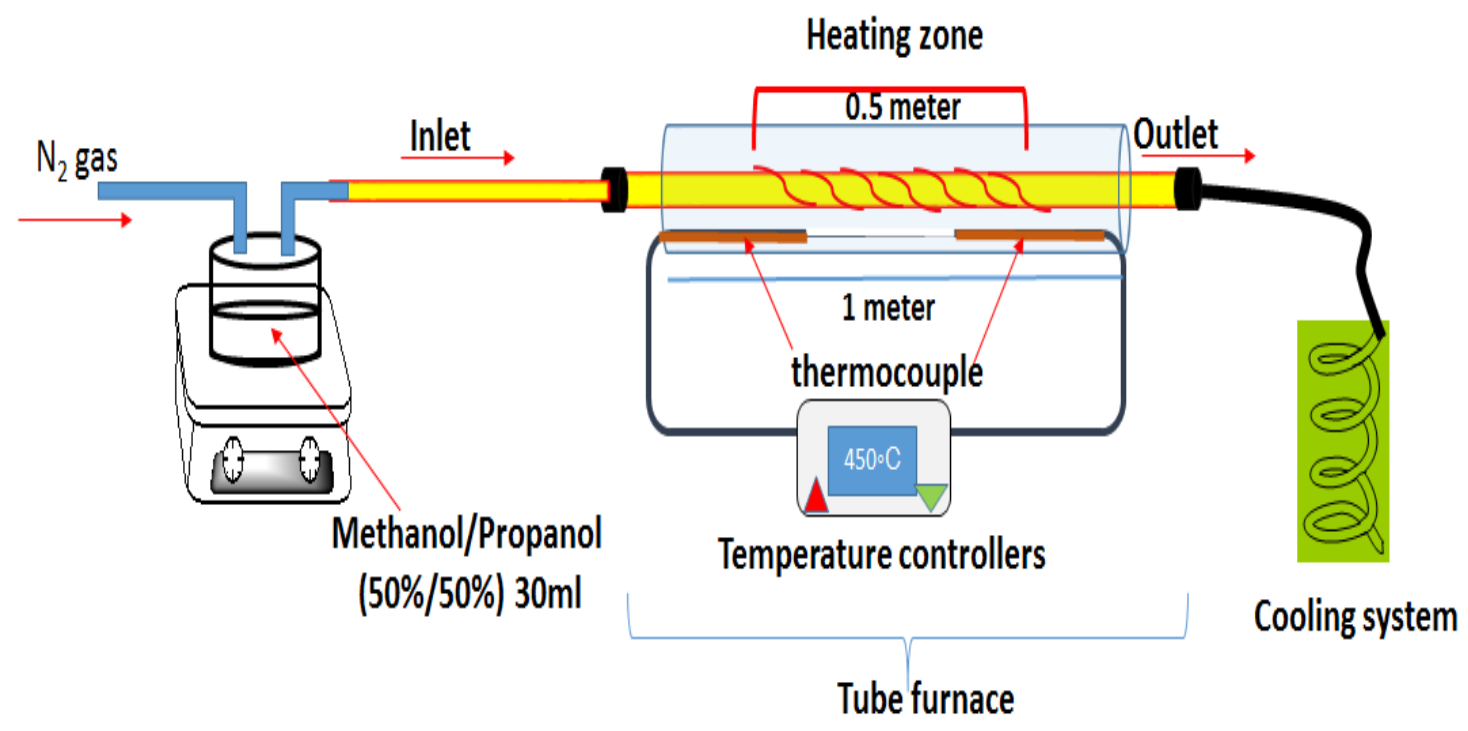

Figure 1. Schematic diagram of tube furnace homemade and reaction system for the synthesis of CNTs by CVD homemade reactor. 
metals such as Fe, Mo, Co, or Ni. The XRD pattern in Figure 2, shows two characteristic peaks the first at $2 \theta \approx 25.48^{\circ}$ and $25.16^{\circ}$, while the second at $43.11^{\circ}$ and $43.78^{\circ}$ for FWNT450 and FWNT750 respectively. The two peaks can be attributed to the diffraction from the $\mathrm{C}(001)$ and $\mathrm{C}(002)$ planes of the CNTs which appears more width and shift to high $2 \theta$ with FWNT450, may relate to more disorder [20]. The second peaks at $2 \theta \approx 43^{\circ}$ were more intense with FWNT750 as compare with FWNT450 which refers to increase the crystalline with increasing temperature. The first peak can be used to calculate inter layer spacing when equal to $0.34 \mathrm{~nm}$ for FWNT750 and $0.35 \mathrm{~nm}$ for FWNT450.

The Raman spectroscopy in Figure 3, shows the D band, which refers to defect style that required to scatter flexible in order to maintain momentum including $\mathrm{sp}^{2}$ and $\mathrm{sp}^{3}$ amorphous carbon. This style is usually located between 1250 and $1450 \mathrm{~cm}^{-1}$ which appear in 1343 and $1362 \mathrm{~cm}^{-1}$ for FWNT750 and FWNT450 respectively [21] The G band were observed at 1596 and $1580 \mathrm{~cm}^{-1}$ for FWNT750 and FWNT450 respectively which variances in intensity with $\mathrm{D}$ band [22]. The intensity of $\mathrm{D}$ to $\mathrm{G}$ bands $\left(\mathrm{I}_{\mathrm{D}} / \mathrm{I}_{\mathrm{G}}\right)$, equal to 1.34 for FWNT450 while that refer to reasonable crystalline quality of FWNT750 when shows 0.76 . Increase the value of $I_{D} / I_{G}$ refers to the distortion on the surfaces of FWNTs that may relate to a low temperature of precipitation [23]. The third weak peaks appear at $2963 \mathrm{~cm}^{-1}$ can be related to overlap with the two peaks $G$ and $D$ on tubular structure which very clear with FWNT750 [24].

Transmission electron microscopy TEM gives a more accurate image of synthesized tubular structure. Figure 3 shows the TEM image

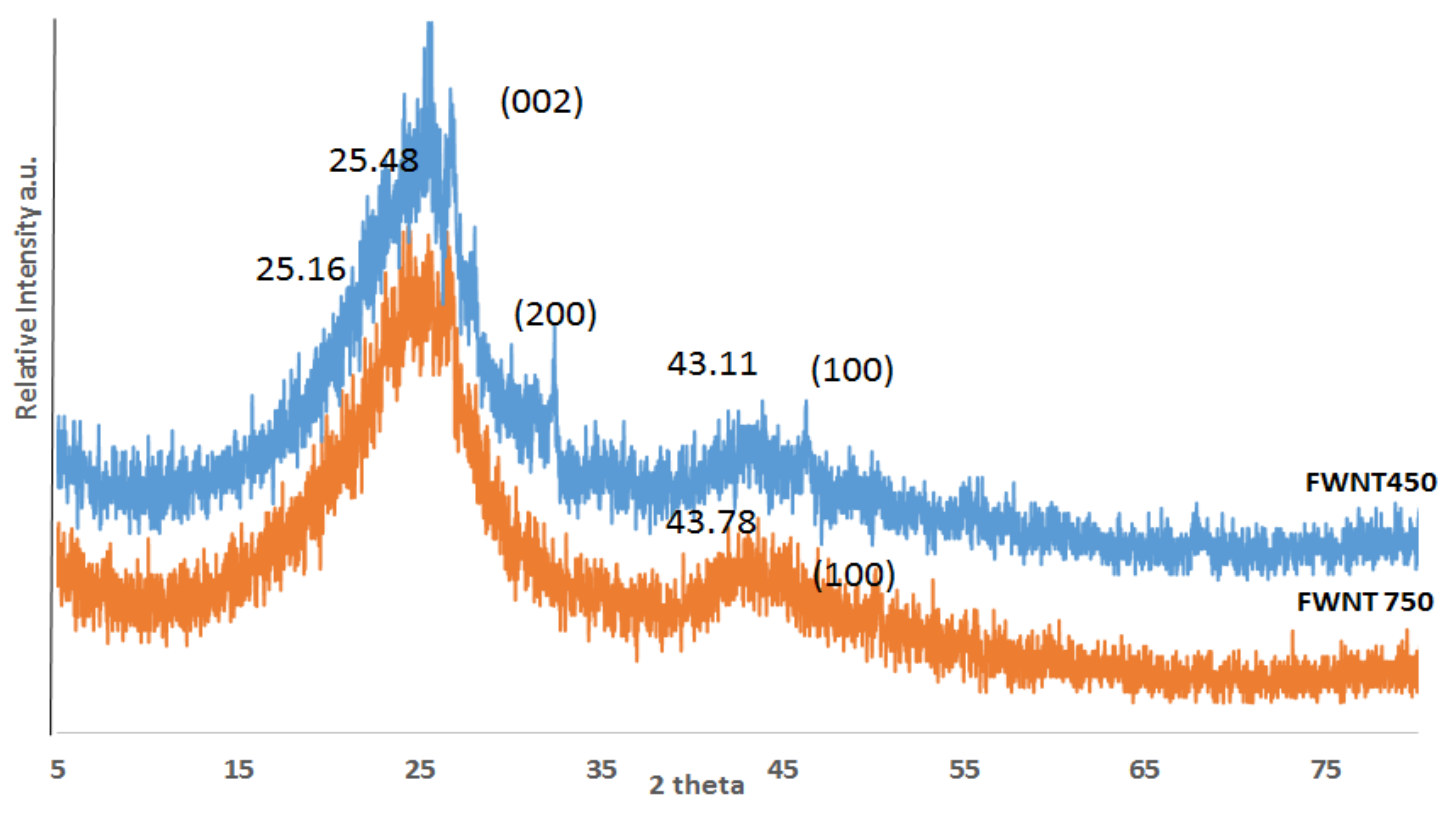

Figure 2. XRD pattern for two types of CNTs which is: FWNT450 and FWNT750 before purification.

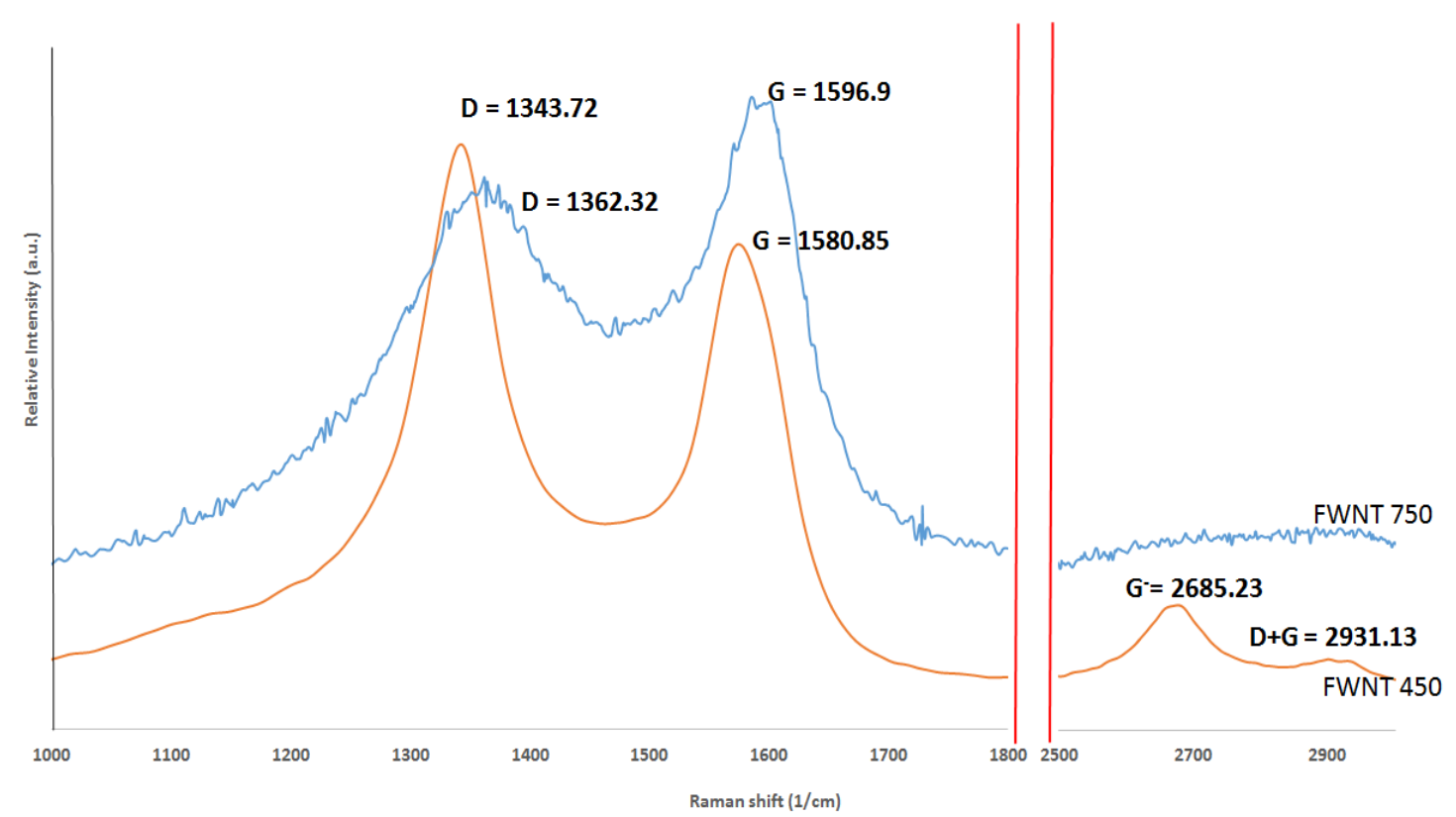

Figure 3. Raman spectroscopy for as prepared FWNT450 and FWNT750. 
of FWNT450, the nanotubes have a range of inner diameters varying from $(\sim 4.21-6.28) \mathrm{nm}$ and outer diameters about $(7.49-11.80) \mathrm{nm}$. The thickness of tubes was 3.28-5.52 nm which refers to 2-4 shells with less regular carbon nanotubes with a long reach to $2 \mu \mathrm{m}$. The TEM images in Figure 4 and Figure 5, shows many particles that can be related to unconverted and amorphous carbon with silica.

TEM image of Figure 5 for FWNT450 shows many distortion on different regains of tubular structure. The distortion which pointed in Figure 5 refer to defect and deformation that forming the tubular structure which increases with decreases temperature of precipitation [16,22].

The condition of synthesized FWNT750 includes high temperature as comparing to FWNT450 which caused more regular for precipitation.
The image of TEM in Fig 6 shows a tubular structure of outer diameter 7.56-10.48 $\mathrm{nm}$ and inner diameter 3.78-5.71 nm. The thickness of tubes was arranged $3.78-4.77 \mathrm{~nm}$ which refers to 3 shells of carbon nanotubes with a length of regular tubes reach to $2 \mu \mathrm{m}$.

The samples were characterized using, Brusquer, Emmett and Teller BET to measure the specific surface area of the materials. The surface area was 279 and $191 \mathrm{~m}^{2} / \mathrm{g}$ for MWNT450 and MWNT750 respectively. The variance in the value of surface area can be related to the variance in the nature of surface as shown in Figure 4 and Figure 6.

The best methods to indicate for this interaction is TGA when the temperature at which carbon nonmaterial is oxidized is an index of their stability [25]. From Figure 7 and Figure8 which explains the

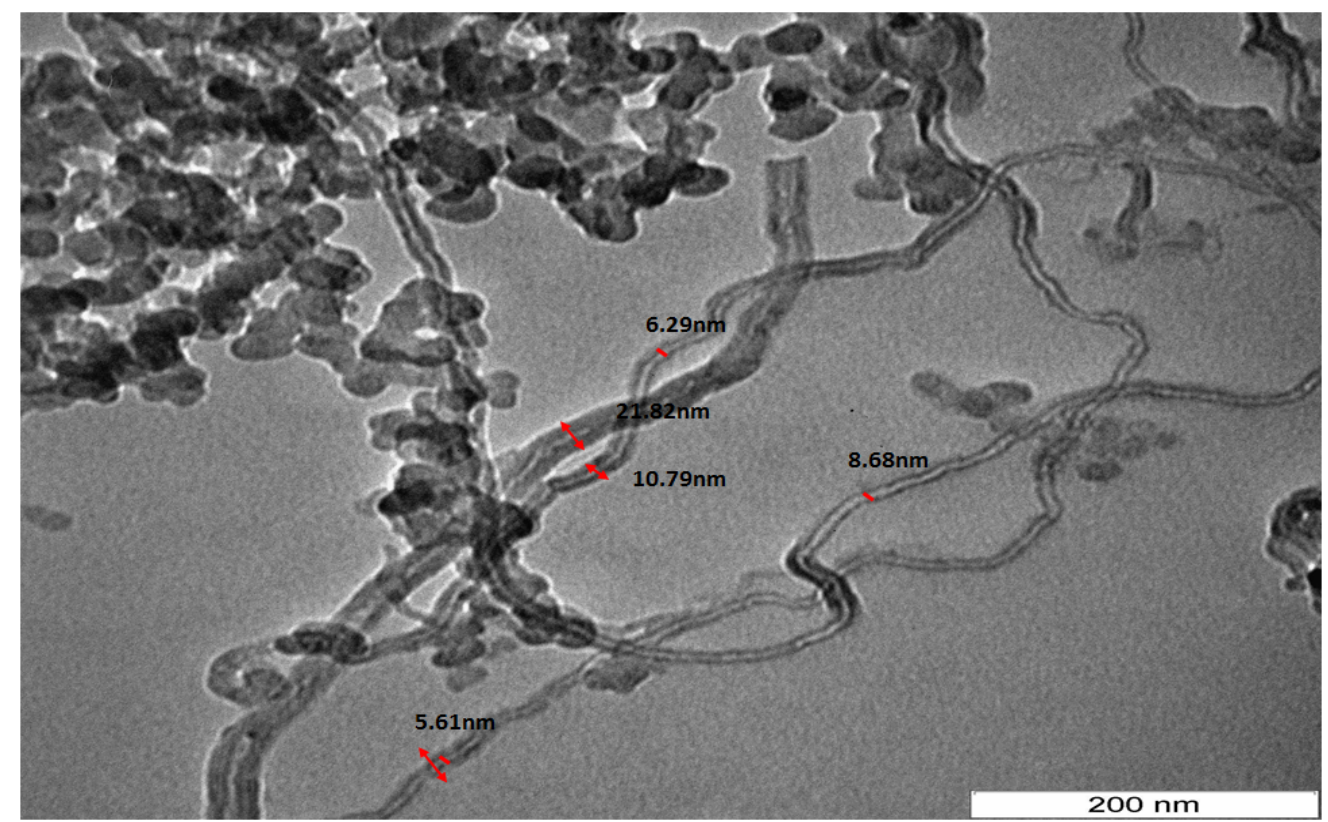

Figure 4. TEM images of as prepared FWNT450 from Methanol/Propanol $(1: 1)$ at $450^{\circ} \mathrm{C}$.

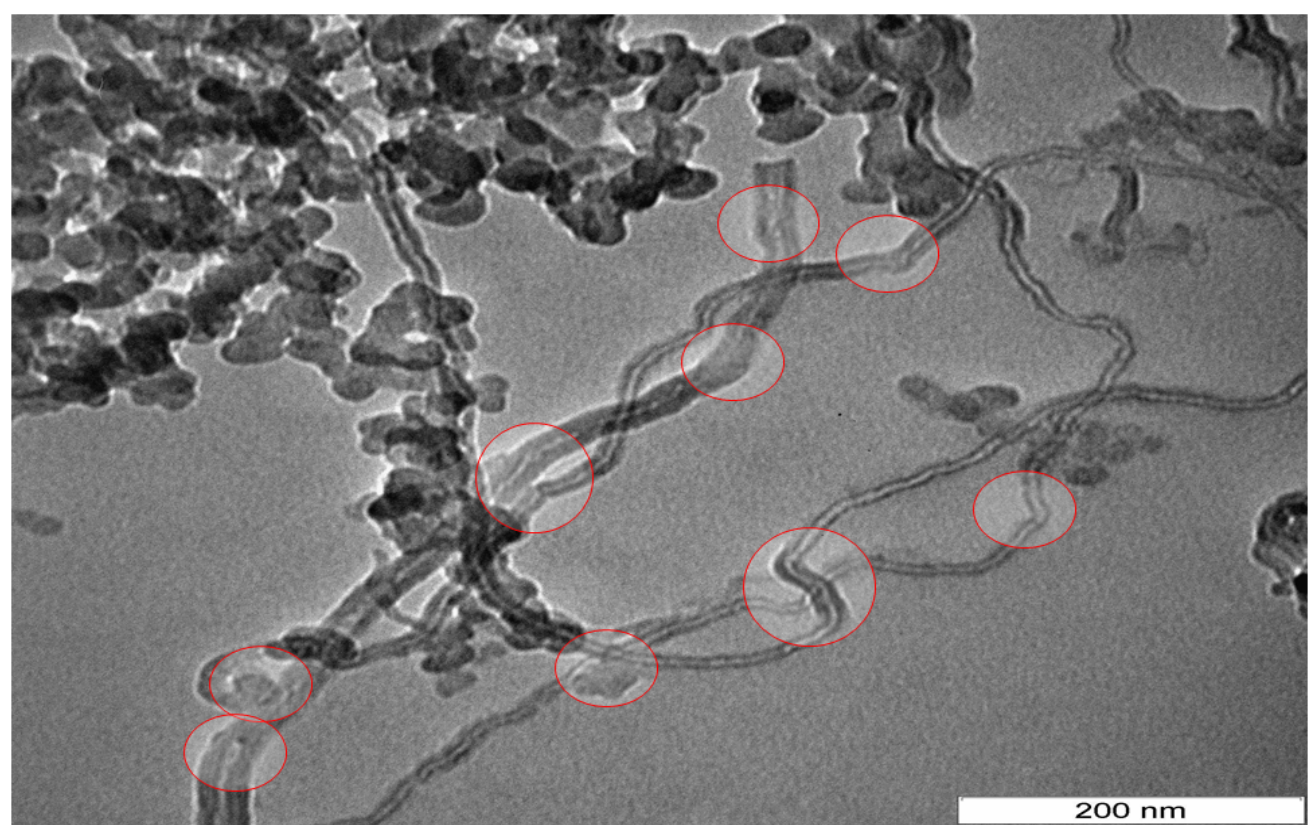

Figure 5. TEM images for synthesized FWNT450 shows many defect and deformation on the surface. 


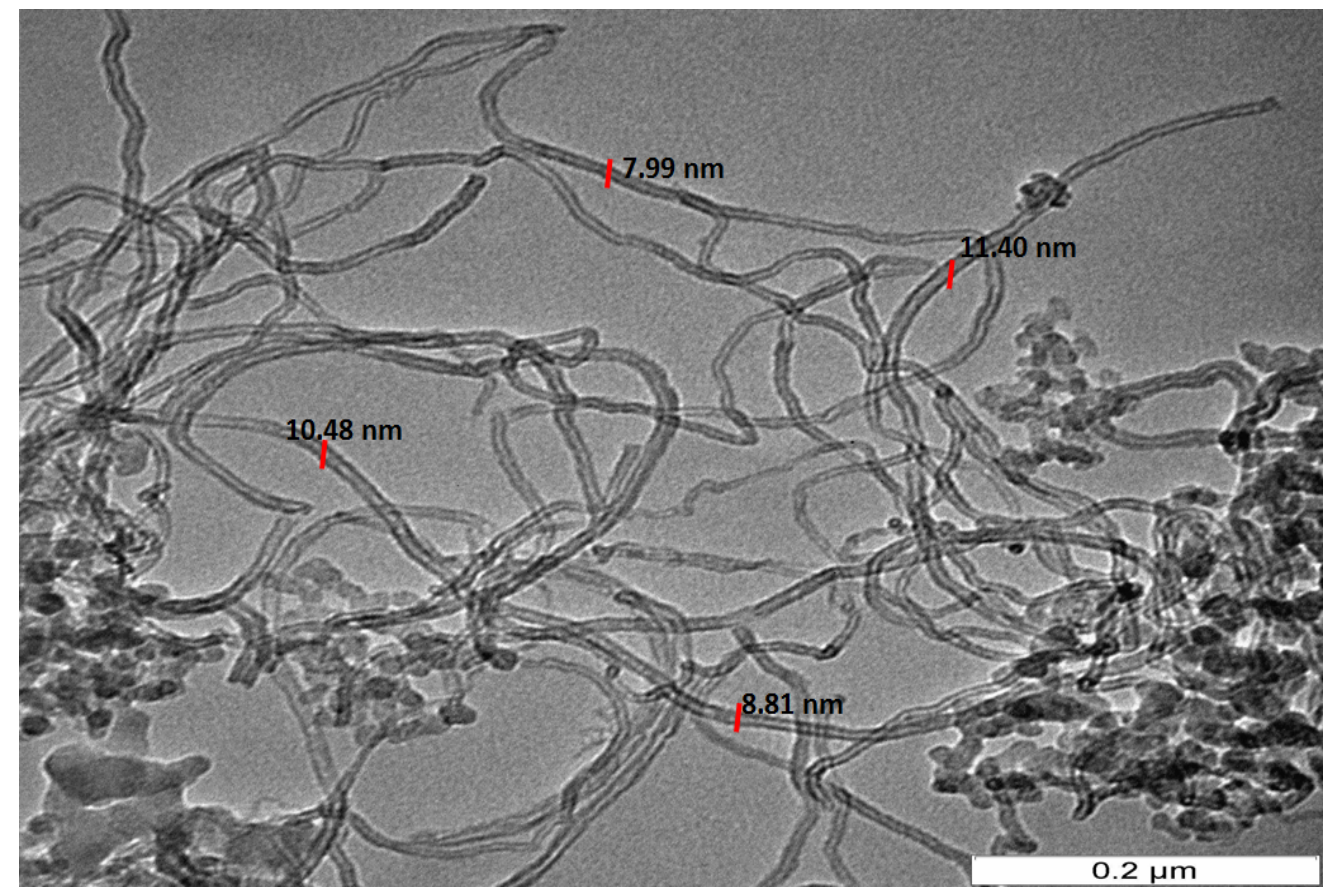

Figure 6. TEM images of as prepared FWNT750.

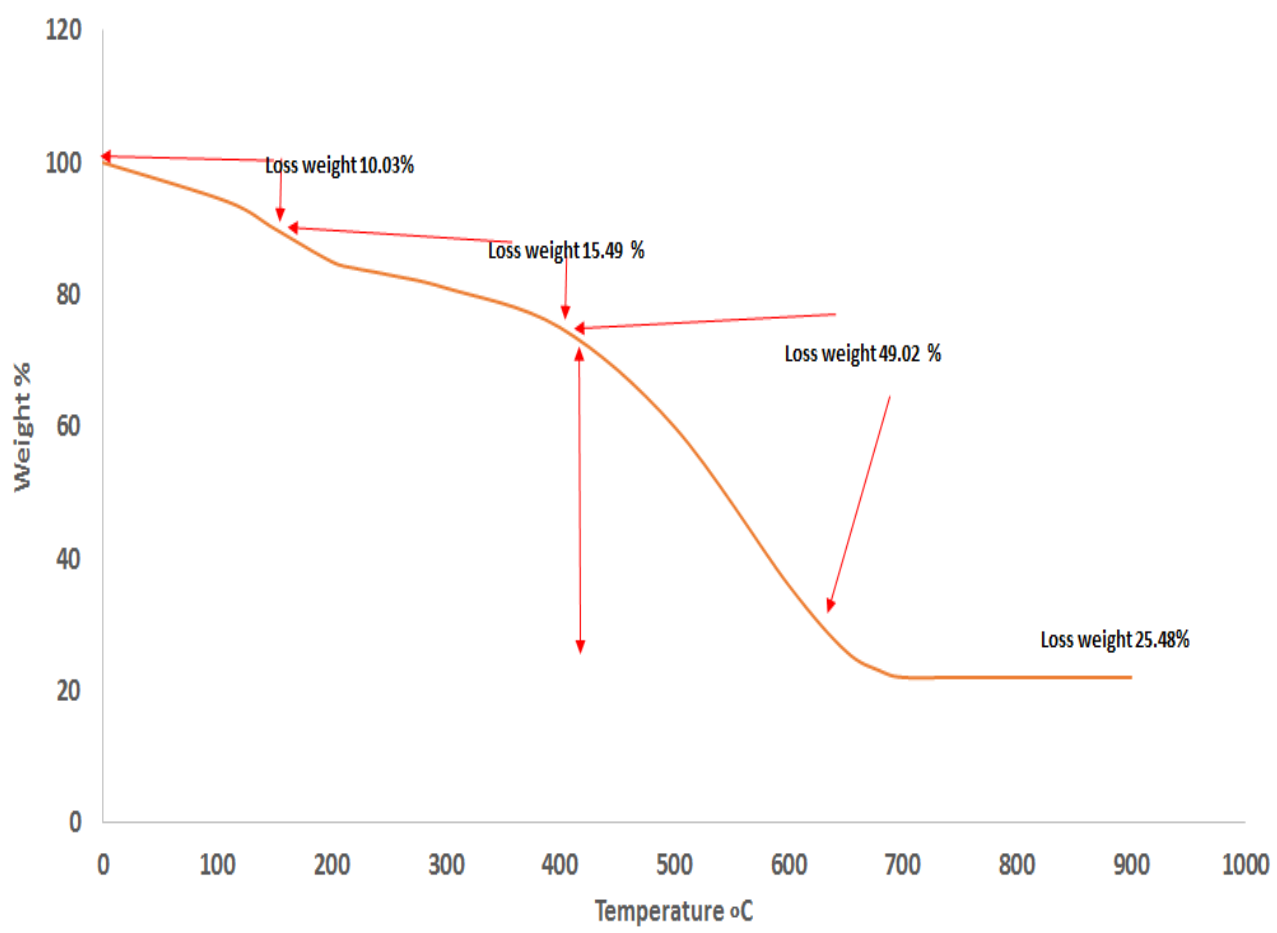

Figure 7. TGA of as-synthesized FWNT450.

identification of synthesized carbon nanotubes with black carbon the before purification. The TGA of FWNT450 includes three steps as shown in Figure7: the first at $\mathrm{T}<100^{\circ} \mathrm{C}$, there is $10.03 \%$ of mass loss that can be attributed to the evaporation of water [26]. The second steps represent by losing $15.49 \%$ on range $100-300^{\circ} \mathrm{C}$ which related to amorphous carbon which appears after more than $150^{\circ} \mathrm{C}$ [27]. The last steps after $430^{\circ} \mathrm{C}$ to $500^{\circ} \mathrm{C}$ refer to decomposition of synthesized FWNT450 [28-29]. While the remaining materials after decomposition all the sample represents $21.99 \%$ mostly attributed to $\mathrm{SiC}$ produce on CVD by reacting the carbon with the support [30]. The ratios of FWNT450 reach to about $49.02 \%$.

Figure 8 shows TGA of FWNT750 which also includes three steps: the first at $\mathrm{T}<100^{\circ} \mathrm{C}$ when $4.18 \%$ loss for adsorption water [26]. The second part from 180 until $470^{\circ} \mathrm{C}$ shows slow oxidation steps causing loss $8.34 \%$ from the sample which refers to unconverted carbon and 


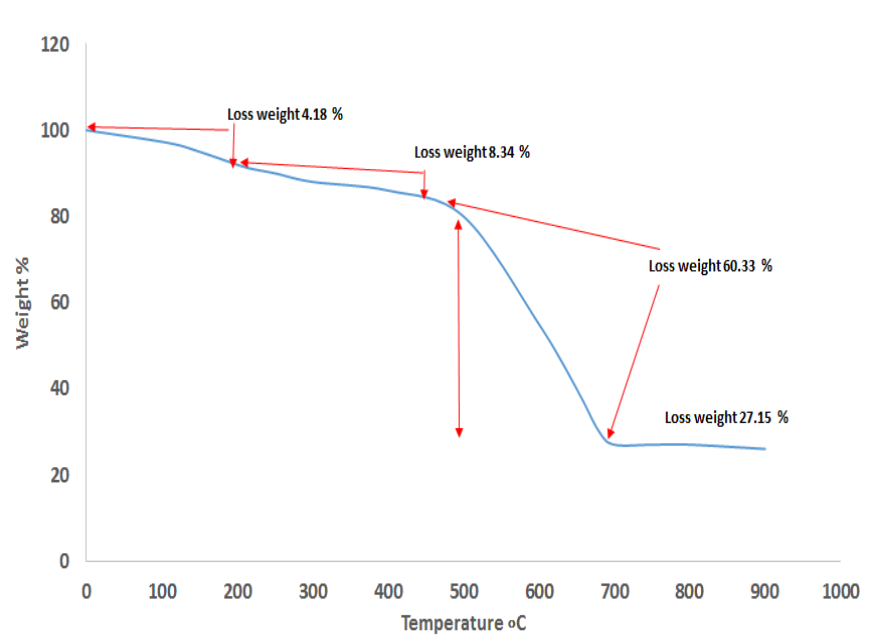

Figure 8. TGA of as-synthesized FWNT750.

amorphous carbon. The ratios $57.67 \%$ refer to FWNT750 when shows rapid oxidation in the part of $500-590^{\circ} \mathrm{C}$ leaving $31.81 \%$ of the residue $\mathrm{SiC}[29,30]$. According to TGA analysis, it can be seen the ratios of amorphous carbon for FWNT450 were more than FWNT750 while thermal stability with the yield of product of FWNT750 which the best as compare with FWNT450 [31].

Luiz et al. [32] reported that both defect and deformation increase the active site on the surface then make the probabilities to make connections with different species easier. Zhang et al. [33] reported that the regular surface of tubular structure reduces the active site and decreases the surface area. Fernando et al. Agreement with Mitri et al. [15] and Raja $\mathrm{R}$ et al. [16] when reported that increase temperature of synthesis CNTs Reduce the defect and deformation which mostly decreases the surface area and active sites [1]. Synthesis of carbon nanotubes with support or catalyst influence directly in purities and types of carbon nanotubes [31]. The influence appears due to the interaction between a catalyst or the support material and the particle size of catalyst $[32,33]$. This reasons influence directly in the number of the wall of product and increased the density of it due to higher density of catalyst and support as comparing with carbon. Furthermore, defect-free CNTs represent highly desirable to limit the application which applied by controlling the conditions of synthesized [33].

\section{Adsorption test}

Measuring the activity of the FWNT450 and FWNT750 was tested by adsorption 40ppm of Congo Red. The typical adsorption experiments of $40 \mathrm{ppm}$ Congo Red with $60 \mathrm{mg}$ of FWNT450 and FWNT750 in aqueous phases. The pH equals to 5 at $298.15 \mathrm{~K}$ which needed for $\lambda_{\max }$ equal to $495 \mathrm{~nm}$ with using a magnetic mixer at 350 $\mathrm{rpm}$. The required amount of the adsorbent where suspend in $100 \mathrm{~cm}^{3}$ of an aqueous solution of Congo Red; at various times $2 \mathrm{ml}$ taken from the reaction vessel, and filtered to remove the particles by centrifuge at 4,000 rpm for 10 minutes in an $800 \mathrm{~B}$ centrifuge. The Congo Red absorbency was measured at $495 \mathrm{~nm}$, using a Shimadzu Cary 100Bio UV - visible spectrophotometer. The activity was tested by measuring the percent of removal and applied the pseudo-first-order kinetics which represents by equation 1 and 2 respectively [34]:

$$
\begin{aligned}
& \text { \% Removal }=\left[\left(\mathrm{C}_{\mathrm{t}}-\mathrm{C}_{0}\right) / \mathrm{C}_{0}\right]^{*} 100 \\
& \ln \left(\mathrm{C}_{0} / \mathrm{C}_{\mathrm{t}}\right)=\mathrm{k}_{1}{ }^{*} \mathrm{t}
\end{aligned}
$$

Where $\mathrm{C}_{0}$ refer to the initial concentration in the bulk solution before starting the adsorption process, $\mathrm{C}_{t}$ refers to concentration of Congo Red after $\mathrm{t}$ min of adsorption. The $\mathrm{k}_{1}$ is the adsorption rate constant. A plot of $\ln \left(\mathrm{C}_{0} / \mathrm{C}_{\mathrm{t}}\right)$ versus $\mathrm{t}$ for Congo Red adsorption with FWNT450 and FWNT750 is presented in Figure 9. The value of $k_{1}$ can be obtained directly from the slope of the respective linear curves in the Figure 10

From Figure 9 and Figure 10, the Removal of Congo Red by FWNT450 increased $10 \%$ and the rate of adsorption was increased $25 \%$ as compare to FWNT750. This result attributed to defect and distortion which increasing the active sites and surface area then caused increasing the adsorbent amount of Congo Red. Many kinds of literature concern with studies the adsorption which explained by function groups or interaction of $\pi$ bonding with different acid or base materials. In this work, the adsorption accrued due increase the surface area with active site causing FWNT450 which more active than FWNT750.

\section{Conclusion}

Few-walled CNTs were prepared by CVD method from a binary mixture of methanol/propanol $(1: 1)$ at $450^{\circ} \mathrm{C}$ and $750^{\circ} \mathrm{C}$ under an $\mathrm{N}_{2}$

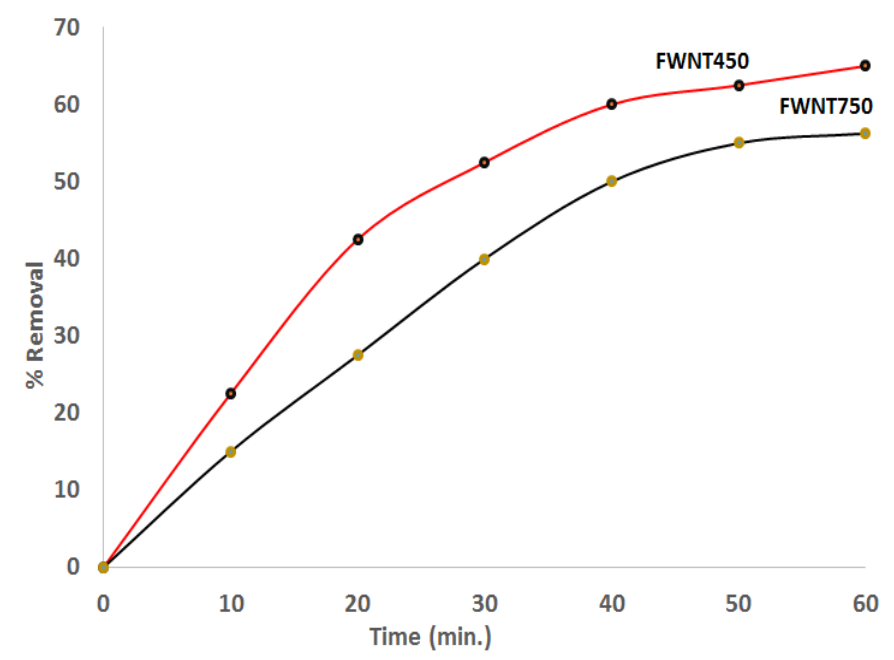

Figure 9. The compare for adsorption 40ppm Congo Red with $60 \mathrm{mg}$ of FWNT450 and FWNT750.

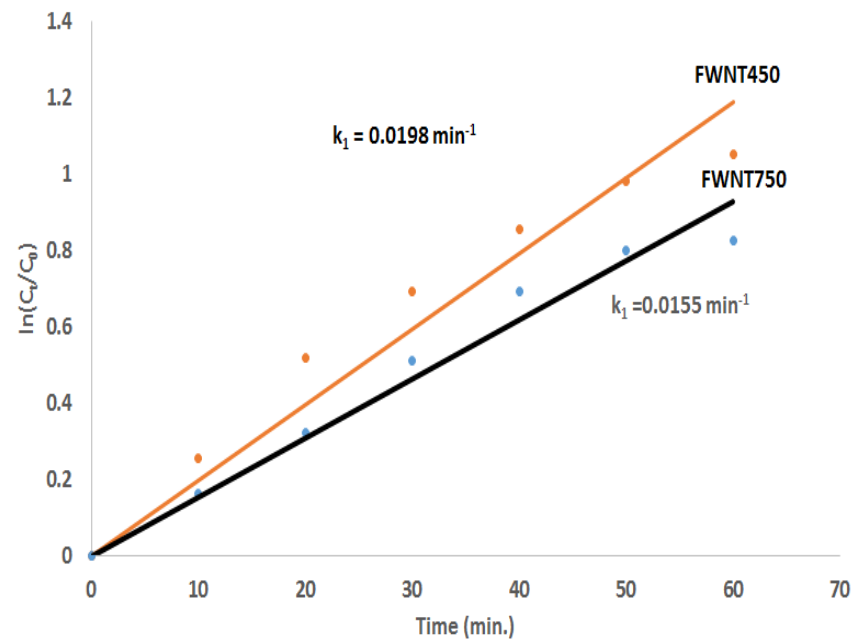

Figure 10. plotting $\ln \left(\mathrm{C}_{\mathrm{t}} / \mathrm{C}_{0}\right)$ vs time for adsorption 40ppm Congo Red with FWNT450 and FWNT750. 
atmosphere on silica and black carbon as support. The process were applied by homemade tube furnace which succeed to synthesized two types of FWNTs in short time and law coast. The images of TEM shows that raising the temperature from $450^{\circ} \mathrm{C}$ to $750^{\circ} \mathrm{C}$ prevent grown the side branching and eliminate the defect or distortion on the surface of the tubular structure. The advantage of the present synthesis technique is synthesized nano-adsorbent materials by simple conditions. The adsorption activity for FWNT450 represent by the conditions of synthesis which forming many distortions on the surface which increase the active site and surface area.

\section{References}

1. Fernando MM, Solange BF, Ivana ZS, Mônica JA (2015) Carbon Nanomaterials as Adsorbents. Springer International Publishing Switzerland 2015.

2. Neil JC, Sabelo DM, Edward NN, Ahmed S (2011) A review of shaped carbon nanomaterials. S Afr J Sci 107: 1-15.

3. Kenan S, Yiying Z, Jiangsha M, Emily C, Navid T, et al. (2013) Structural polymerbased carbon nanotube composite fibers: Understanding the processing structure performance relationship. Materials 6: 2543-2577.

4. Cheng Q, Hang Q, Bo G, Yuan C, Qi Q et al. (2006) Fabrication of Small Diameter Few-Walled Carbon Nanotubes with Enhanced Field Emission Property. J Nanos Nanotechn 6: 1346-1349. [Crossref]

5. Andrea S, Caterina P, Anita C, Girolamo G, Danilo V, et al. (2010) Synthesis Methods of Carbon Nanotubes and Related Materials. Materials 3: 3092-3140.

6. Satiskumar BC, Govindaraj A, Rao CNR (1999) Bundles of aligned carbon nanotubes obtained by the pyrolysis of ferrocene-hydrocarbon mixtures: role of the metal nanoparticles produced in situ. Chemi Phys Let 307: 158-162.

7. Li WZ, Xie SS, Qian LX, Chang BH, Zou BS, et al. (1996) Large-Scale Synthesis of Aligned Carbon Nanotubes. Science 274: 1701-1703. [Crossref]

8. Sen R, Govindaraj A, Rao C (1997) Carbon Nanotubes by the metallocene route. Chem Phys Let 267: 276-280.

9. Li N, Chen X, Stoica L, Xia W, Qian, J, et al. (2007) The catalytic synthesis of threedimensional hierarchical carbon nanotube composites with high electrical conductivity based on electrochemical iron deposition. Adv Mate 19: 2957-2960.

10. Hang Q, Cheng Q, Jie L (2006) Synthesis of High-Purity Few-Walled Carbon Nanotubes from Ethanol/Methanol Mixture. Chem Mater 18: 5691-5695.

11. Vander R, Tichich M, Curtis V (2001) Substrate-support Interactions in MetalCatalyzed Carbon Nanofiber Growth. Carbon 39: 2277-2289.

12. Reilly P, Whitten W (2006) The Role of Free Radical Condensates in the Production of Carbon Nanotubes during the Hydrocarbon CVD Process. Carbon 44: 1653-1660.

13. Aguilar EA, Antúnez W, Alonso G, Delgado FP, Magaña FE, et al. (2006) Study of carbon nanotubes synthesis by spray pyrolysis and model of growth. Diam Relat Mater 15: $1329-1335$.

14. Andrea S, Caterina P, Anita C, Girolamo G, Danilo V et al. (2010) Synthesis Methods of Carbon Nanotubes and Related Materials. Mate 3: 3092-3140.

15. Mitri S, Sotrirchos S (2005) Production of Carbon Nanotubes using CVD- Study of the Operating Parameters. J Rev Adv Mate Sci 10: 314-319.
16. Rajarad R, Ramachndra B (2012) Study of effect of temperature on the synthesis of carbon nanotubes by floating catalyst method. J Met Mat Main 22: 1-5.

17. Nady AF (2017) Carbon nanotubes synthesis using carbonization of pretreated rice straw through chemical vapor deposition of camphor. RSC Adv 7: 28535-28541.

18. Kure N, Hamidon MN, Azhari S, Mamat NS, Yusoff HM, et al. (2017) Simple Microwave-Assisted Synthesis of Carbon Nanotubes Using Polyethylene as Carbon Precursor. J Nanomat Article ID 2474267: 4.

19. Gonz'alez IZ, Valenzuela AM, Alonso G, Far'ias MH, G'omez YV (2017) Influence of the Synthesis Parameters in Carbon Nanotubes Doped with Nitrogen for Oxygen Electro reduction. ECS Sol St Sci Tech 6: 3135-3139.

20. Firas HA, Shahad KE, Halimah AD, Ahmed MA, Mustafa KAK (2016) X-ray Analysis for Purification Process of Synthesized Multi-Walled Carbon Nanotubes by Chemical Vapor Deposition. Inte J Theo Appl Scie 8: 37-43.

21. Dilip KS, Iyer PK, Giri PK (2010) Diameter dependence of interwall separation and strain in multiwalled carbon nanotubes probed by X-ray diffraction and Raman scattering studies. Diam Rel Mat 19: 1281-1288.

22. Ferrari AC, Robertson J (2001) Resonant Raman spectroscopy of disordered, amorphous, and diamond-like carbon. Phys Rev B 64: 75414-75427.

23. Park Y, Choi Y, Kim K, Chung D, Bae D et al. (2001) High yield purification of multiwalled carbon nanotubes by selective oxidation during thermal annealing. Carbon 39: 655-61.

24. Dikio ED, Shooto ND, Thema FT, Farah AM (2013) Raman and TGA Study of Carbon Nanotubes Synthesized Over Mo/Fe Catalyst on Aluminium Oxide, Calcium Carbonate and Magnesium Oxide Support. Chem Sci Trans 2: 1160-1173.

25. Datsyuk V, Kalyva M, Papagelis K, Parthenios J, Tasis D, et al. (2008) Chemical oxidation of multiwalled carbon nanotubes. Carbon 46: 833- 840 .

26. Elsa GOC, Manuel RA, Alfredo AE, Francisco EM (2013) Synthesis of Carbon Nanotubes of Few Walls Using Aliphatic Alcohols as a Carbon Source. Mater 6: 2534-2542.

27. Shajahan M, Mo Y, Nahm K (2003) Low cost growth route for single-walled carbon nanotubes from decomposition of acetylene over magnesia supported Fe-Mo catalyst. Kor J Chem Eng 20: 557-566.

28. Feng Y, Zhang H, Hou Y, McNicholas TP, Yuan D, et al. (2008) Room temperature purification of few-walled carbon nanotubes with high yield. ACS Nano 2: 1634-1638. [Crossref]

29. Zhenyu R, Jingtang Z, Maozhang W, Bijiang Z (2002) Preparation and characterization of silicon carbide fibers from activated carbon fibers. Carbon 40: 715-720.

30. Kao C, Young R (2004) A Raman spectroscopic investigation of heating effects and the deformation behavior of epoxy/SWNT composites. Comp. Scie. Tech. 64, 2291-2295.

31. Costa S, Palen E, Kruszynsky M, Bachimatiuk A, Kalenczuk R (2008) Characterization of carbon nanotubes by Raman spectroscopy. Mate Scie Poland 26: 433-441.

32. Luiz A, Anna CD, Marcelo BP, Flavio H, Carlos PB (2016) Influence of Different Defects in Vertically Aligned Carbon Nanotubes on $\mathrm{TiO}_{2}$ Nanoparticle Formation through Atomic Layer Deposition. Aces Appl Mater Interfaces 8: 16444-16450.

33. Rufan Z, Yingying Z, Fei W (2017) Controlled Synthesis of Ultralong Carbon Nanotubes with Perfect Structures and Extraordinary Properties. Acc Chem Res 50: 179-189.

34. Tariq AM, Firas HA (2016) Oxidation of multi-walled carbon nanotubes in acidic and basic Piranha mixture Furi Nanosc Nanotech 2: 155-158.

Copyright: (C2017 Abdulrazzak FH. This is an open-access article distributed under the terms of the Creative Commons Attribution License, which permits unrestricted use, distribution, and reproduction in any medium, provided the original author and source are credited. 